EPJ Web of Conferences 67, 02062 (2014)

DOI: $10.1051 /$ epjconf / 20146702062

(C) Owned by the authors, published by EDP Sciences, 2014

\title{
Ventilation of idealised urban area, LES and wind tunnel experiment
}

\author{
L. Kukačka ${ }^{1,2, a}$, V. Fuka ${ }^{1}, \check{S}$. Nosek ${ }^{2}$, R. Kellnerová ${ }^{1,2}$, and Z. Jaňour ${ }^{2}$ \\ ${ }^{1}$ Charles University in Prague, Faculty of Mathematics and Physics, The Department of Meteorology and Environment \\ Protection, Czech Republic \\ 2 Institute of Thermomechanics Academy of Sciences of the Czech Republic, v.v.i, Dolejškova 1402/5, Prague 182 00, \\ Czech Republic
}

\begin{abstract}
In order to estimate the ventilation of vehicle pollution within street canyons, a wind tunnel experiment and a large eddy simulation (LES) was performed. A model of an idealised urban area with apartment houses arranged to courtyards was designed according to common Central European cities. In the wind tunnel, we assembled a set-up for simultaneous measurement of vertical velocity and tracer gas concentration. Due to the vehicle traffic emissions modelling, a new line source of tracer gas was designed and built into the model. As a computational model, the LES model solving the incompressible Navier-Stokes equations was used. In this paper, we focused on the street canyon with the line source situated perpendicular to an approach flow. Vertical and longitudinal velocity components of the flow with the pollutant concentration were obtained from two horizontal grids placed in different heights above the street canyon. Vertical advective and turbulent pollution fluxes were computed from the measured data as ventilation characteristics. Wind tunnel and LES data were qualitatively compared. A domination of advective pollution transport within the street canyon was determined. However, the turbulent transport with an opposite direction to the advective played a significant role within and above the street canyon.
\end{abstract}

\section{Introduction}

Vehicle traffic became often a dominant pollution source in large cities all over the world [1]. Air quality improvement in urban areas is necessary to avoid risk for human health [2]. The problem of the pollution transport within the built-up areas were summarised in several recent works [3-5]. A mass exchange between street canyon and external atmospheric flow and the influence of the external turbulence intensity on the scalar transfer from the canyon was investigated in [6]. A pollution transfer within a model of a real street intersection in Central London (DAPPLE series of projects) was balanced in [7]. Finally, the investigation of advective and turbulent fluxes within street intersections in a complex urban area was presented in [8]. We used similar experimental method in the present paper. A significant role of a building geometry influence on the flow patterns and pollutant dispersion was proved in [9]. Highly three-dimensional flow and an important role of a turbulent pollution exchange at roof level above the street intersections was demonstrated in $[8,10]$. Mechanisms of the turbulent mass transport above the street intersection were subsequently analysed in [11].

In this study, we have followed our recent research described in $[10,11]$. We present a part of the complex project that aims to investigate the influence of the building height variation and the ground arrangement on the ventilation intensity. Presented experiment and numerical simulation are focused on vertical advective and turbulent pollution transport above a finite street canyon within the one type

\footnotetext{
a e-mail: kukacka@it.cas.cz
}

of an idealised built-up area. Understanding processes of pollution transport in complex urban areas could enable better estimation of the ventilation intensity within street canyons. Consequently, this could provide opportunities for designing suitable configuration of built-up areas and effective development of local scale dispersion models.

\section{Experimental set-up}

\subsection{Urban area model}

The model was designed according to an idealised urban area formed by apartment houses with pitched roofs arranged in courtyards that can be found in central Prague. The model was manufactured in scale 1:400, see figure 1 .

The height of model buildings with pitched roof was $H=62.5 \mathrm{~mm}$ ( $25 \mathrm{~m}$ in full scale $)$. The width of street canyons was $S=50 \mathrm{~mm}$ ( $20 \mathrm{~m}$ in full scale). The aspect ratio of the street canyons was $H / S=1.25$.

\subsection{Wind tunnel experiment}

\subsubsection{Wind tunnel}

The physical experiment was performed in the open lowspeed wind tunnel of Institute of Thermomechanics Academy of Sciences of the Czech Republic in Nový Knín. The cross dimension of the tunnel was $1.5 \times 1.5 \mathrm{~m}$. The development section of the tunnel was $20.5 \mathrm{~m}$ long and the length of the test section was $2 \mathrm{~m}$. The scheme of the tunnel is depicted in figure 2. 


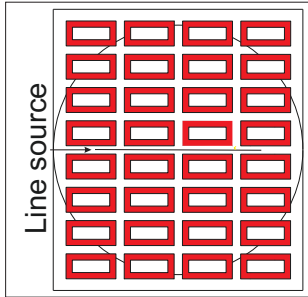

(a)

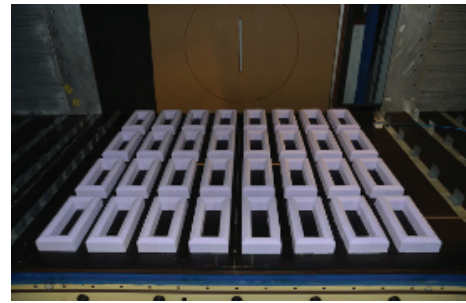

(b)

Fig. 1. Scheme of the idealised symmetric urban area model (a) and the photograph of the model placed in the wind tunnel (b).

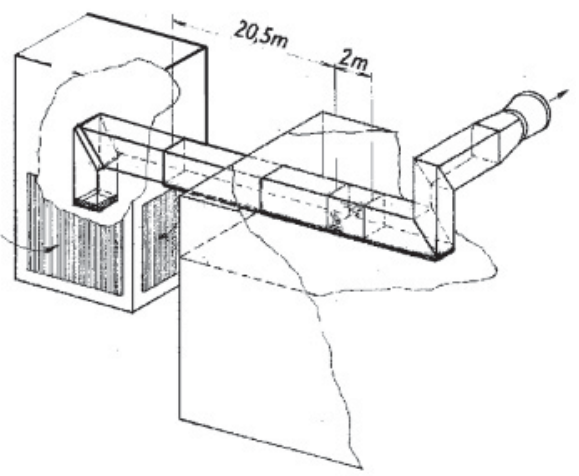

Fig. 2. The scheme of the open low-speed wind tunnel.

\subsubsection{Line source of tracer gas}

A new line source of a tracer gas was designed and manufactured for the urban area model according to the vehicle traffic pollution simulation. The total length of the line source was $L=1 \mathrm{~m}$ (400 $\mathrm{m}$ in full scale). The $1 \mathrm{~m}$ long line source was formed by four independent units. In every $250 \mathrm{~mm}$ long unit, a line of 126 equally spaced needles was built in. Needles with inner diameter $0.3 \mathrm{~mm}$ were 100 $\mathrm{mm}$ long. The pressure drop across the needles exceeded the stagnation pressure in the wind tunnel of $130 \mathrm{~Pa}$. That was sufficiently high value in comparison with expected pressure fluctuations on the tunnel floor (up to $10 \mathrm{~Pa}$ ). The line source homogeneity was successfully verified before measurement.

\subsubsection{Measurement techniques}

A simultaneous point measurement of hydrocarbon concentration together with longitudinal and vertical velocity components was realised by Fast-response Flame Ionisation Detector (FFID) with two-dimensional optical fibre Laser Doppler Anemometry (LDA). LDA and FFID probes were mounted on the traverse system in a way that the measuring volume of the LDA was close to the intake to the FFID sampling tube, see Figure 3. A detailed description of the measurement can be found in [11].

We used ethane as the tracer gas simulating passive pollutants emitted by vehicle traffic. A data rate of LDA reached about $200 \mathrm{~Hz}$ in a measured area; the FFID detector was set to acquire data at the data rate of $1 \mathrm{KHz}$. A freestream velocity of the flow in the tunnel was measured using
Prandtl tube fixed in the centre of tunnel cross-section $4 \mathrm{~m}$ upwind from the test section.

We applied Matlab post-processing script to synchronise the simultaneously acquired vertical velocity and concentration data finding a maximum correlation between signals. The synchronised data were shifted by an average of $12 \mathrm{~ms}$. This shift expressed the delay between the suck of the sample into the intake of the FFID probe tube and the moment of the sample analysing in the probe. The value of the shift agrees with very similar experimental set published in recent works $[11,12]$.

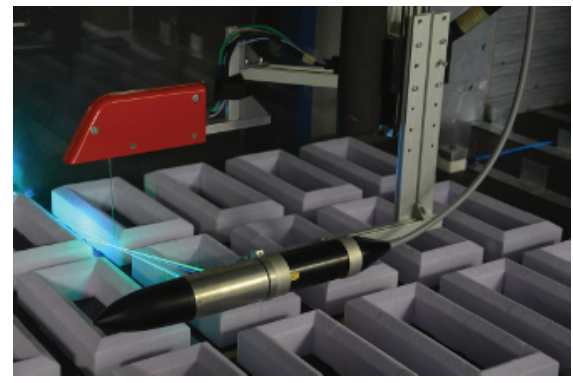

(a)

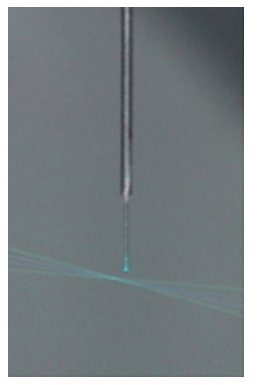

(b)
Fig. 3. The configuration of the FFID (red) and LDA (black and silver) probes mounted on the traverse system in the wind tunnel (a). The detail of the measuring volume of LDA with FFID sampling tube intake (b).

\subsection{Large eddy simulation}

\subsubsection{Numerical model}

A numerical part of this work was computed by CLMM model (Charles University Large-eddy Microscale Model), for further details see [13].

The model utilised large eddy simulation (LES) for the turbulent flow. Navier-Stokes equations were solved in incompressible form by the fractional step method in the model. Equations are discretized using the third order Runge-Kutta and the semi-implicit Crank-Nicolson methods.

The finite volume method on a uniform Cartesian grid was used in space. The spatial discretization of the fluxes was the second order central except. The momentum advection and scalar advection that were solved by the fourth order central and the third order positive method [14], respectively. The nonlinear terms are filtered with filter width equal to two cell widths [15]. The sigma subgrid model [16] is utilised in subfilter (subgrid) scales computations.

\subsubsection{Boundary conditions}

The above described simulation was carried out with the model at the scale of 1:400 and finite line source according to the wind tunnel experiment. The boundary conditions were set-up periodic on horizontal domain boundaries. The flow was driven by a constant pressure gradient. The top boundary was a zero stress (free-slip) wall. 


\subsection{Boundary layer characteristics}

A fully turbulent boundary layer (BL) was formed by development section of the tunnel. At the beginning of this section, three triangular turbulent generators were placed there. The floor of the section was covered by roughness elements of dimensions 50 x $50 \mathrm{~mm}$.

The turbulent characteristics of the BL were measured with a two-dimensional LDA system in four vertical profiles placed in different positions among roughness elements around $330 \mathrm{~mm}$ upwind from the model, see the scheme in figure 4.

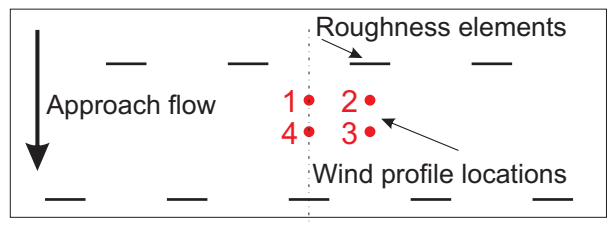

Fig. 4. Wind profile measurement locations.

The vertical profile of mean longitudinal velocity is depicted in figure 5a, the momentum flux profile can be found in figure $5 \mathrm{~b}$. The vertical profiles of longitudinal and vertical turbulent intensity are plotted in figures $5 \mathrm{c}$ and $5 \mathrm{~d}$. The height above the surface is expressed in full scale.

A representative vertical profile of longitudinal mean velocity no. 4 was fitted by the logarithmic and the power law. Mean roughness length $z_{0}$, displacement $d_{0}$ and friction velocity $u_{*}$ (alias square-root of constant Reynolds stress within the inertial sublayer) were obtained from the log wind profile fitting. Power exponent $\alpha$ was obtained from the power wind profile fitting. The BL parameters are listed in table 1. According to VDI guideline [17], modelled BL

Table 1. Parameters of the wind tunnel boundary layer (in full scale).

\begin{tabular}{cccc}
\hline$z_{0}(m)$ & $d_{0}(m)$ & $\alpha(-)$ & $u_{*} / U_{\text {FreeStream }}(-)$ \\
\hline 1.87 & 2.96 & 0.27 & 0.07 \\
\hline
\end{tabular}

met the requirements of the flow above densely built-up inner-city area with a small building height variation. The vertical wind profile no. 4 was used as a starting condition of the flow in the LES.

To verify requirements for the Townsend hypothesis [18], an independency on Reynolds building number $R_{B}$ was verified for measured quantities. In our experiment, the modified Reynolds building number was given by

$$
\operatorname{Re}_{B}=\frac{U_{2 H} H}{v},
$$

where $U_{2 H}$ is reference longitudinal velocity measured at a height of $z=2 H$ and $v$ is kinematic viscosity. This criterion is usually used for the flow within street canyons to be independent of viscous effects, see $[19,20]$.
The experiment was carried out by $R e_{B} \approx 9000$ that lied on a lower edge of the determined interval for valid Townsend hypothesis and corresponded to the free stream velocity $U_{\text {FreeStream }} \approx 3.5 \mathrm{~m} \mathrm{~s}^{-1}$ and to the reference velocity $U_{2 H} \approx 2.0 \mathrm{~m} \mathrm{~s}^{-1}$.

\section{Results}

\subsection{Area of the results}

In the wind tunnel, the data were measured in two horizontal planes at the level $\mathrm{z} / \mathrm{H}=0.8$ (at eaves height) and $\mathrm{z} / \mathrm{H}=$ $1.2 \mathrm{H}$ (above the roof) above the street canyon with the line source, see the scheme in Figure 6. This street canyon was oriented perpendicular to the approach flow.

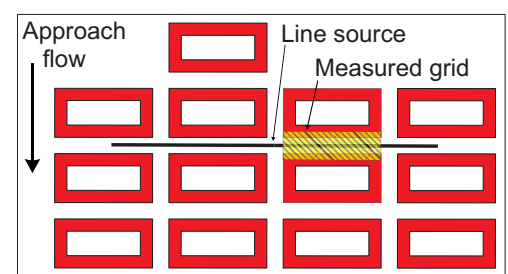

(a)

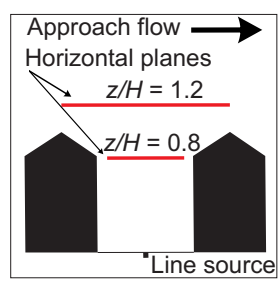

(b)
Fig. 6. Location of measured grids above the street canyon: top view (a) and side view (b).

For LES simulation, a computational domain was situated in a windward half of the model. The domain, containing $161 \times 281 \times 101$ grid points, was set-up with the resolution of $5 \mathrm{~mm}$ ( $2 \mathrm{~m}$ in full scale). Simulation results are displayed for a middle part of the domain, see Figure 7.

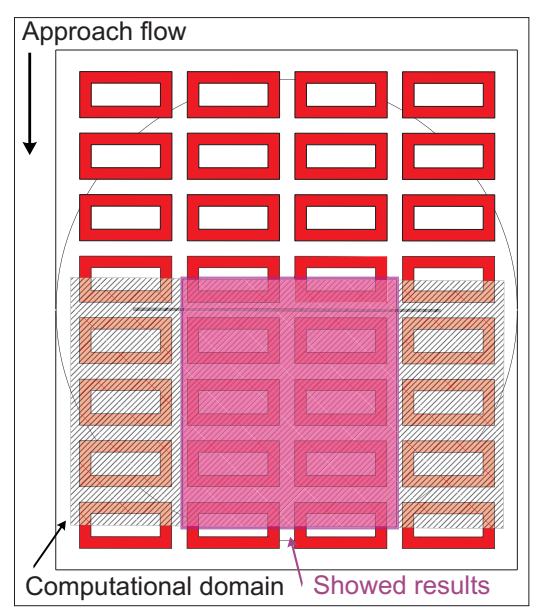

Fig. 7. The scheme of the computational domain of LES simulation (hatched) and results area (coloured).

\subsection{Quantities definition}

The data from the wind tunnel experiment and the LES simulation were transformed to dimensionless quantities 


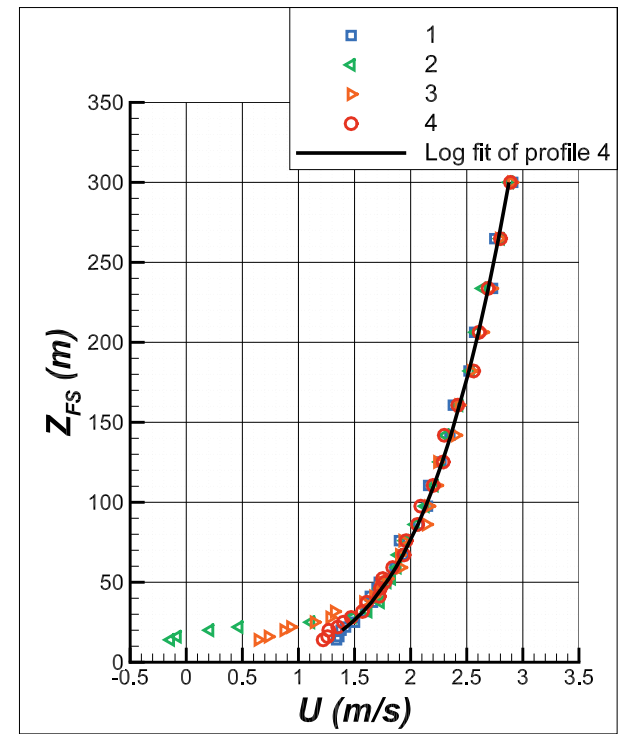

(a) The vertical profiles of mean longitudinal velocity.

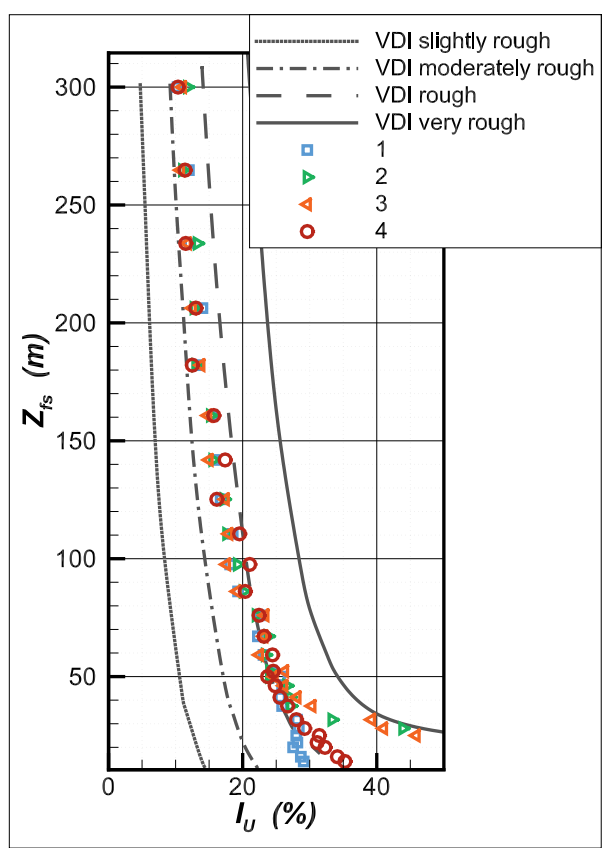

(c) The vertical profiles of longitudinal turbulent intensity with upper bounds of VDI profiles for various roughness classes [17].

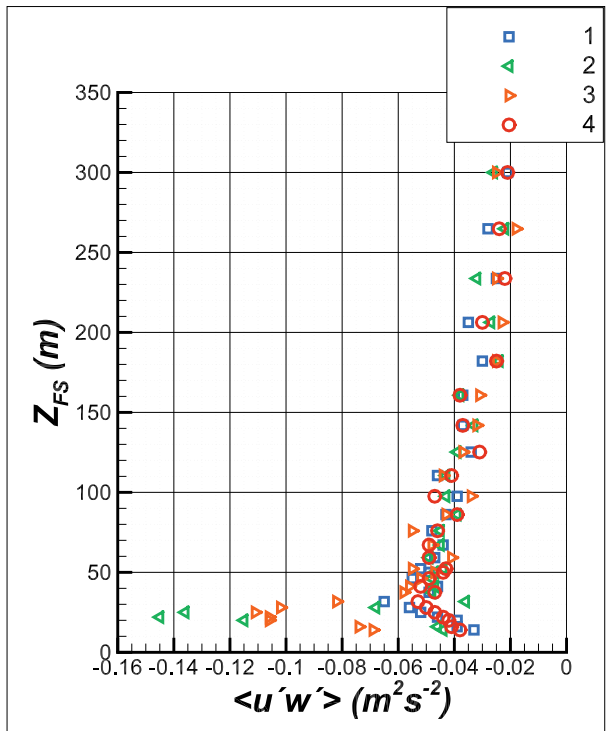

(b) The vertical profiles of mean momentum flux.

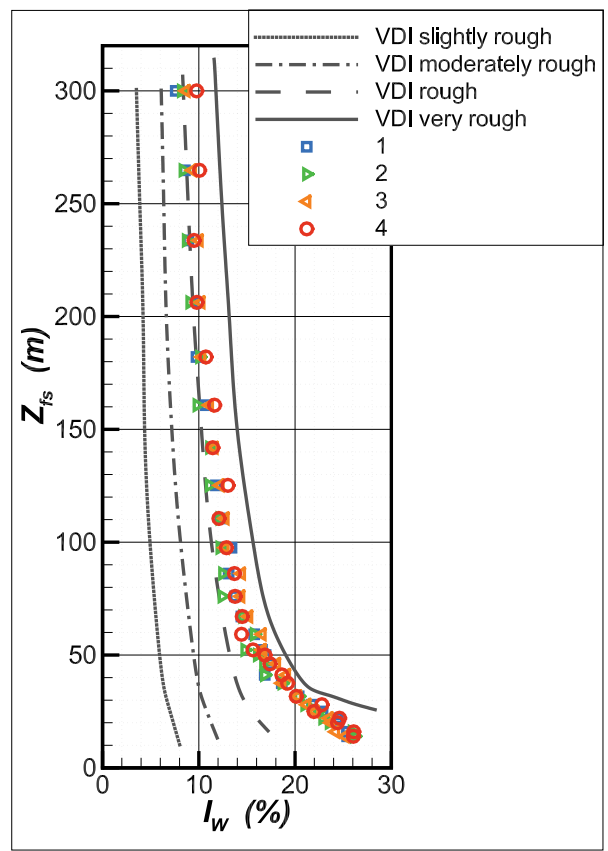

(d) The vertical profiles of vertical turbulent intensity with upper bounds of VDI profiles for various roughness classes [17].

Fig. 5. Characteristics of the approach boundary layer in the wind tunnel.

using following formulas. Longitudinal $U$ and vertical $W$ velocity components were converted to dimensionless form as

$$
\frac{U}{U_{2 H}}, \frac{W}{U_{2 H}},
$$

where $U_{2 H}$ is is the reference longitudinal velocity measured at the height of $z=2 \mathrm{H}$ above the middle of the model.
The dimensionless concentration of the tracer gas $C^{*}$ was given as

$$
\frac{C U_{2 H} H L}{Q}
$$

where $C$ means the measured volume concentration, $L$ is the line source length and $Q$ is the source emission volume flow [17].

We computed the dimensionless vertical advective pollution flux as

$$
\frac{C^{*} W}{U_{2 H}}
$$


The dimensionless vertical turbulent pollution flux was defined as

$$
\frac{c^{* \prime} w^{\prime}}{U_{2 H}}
$$

where \langle\rangle is the time average, $c^{* \prime}$ is the dimensionless concentration fluctuation and $w^{\prime}$ indicated the fluctuation of the vertical velocity (e.g. $[11,8]$ ).

In a vertical direction, the positive and negative signs mean the movement outwards and inwards the street canyon, respectively.

\subsection{Velocity fields}

Approximately symmetrically distributed data of the dimensionless longitudinal and vertical velocities were measured in both horizontal planes above the street canyon, see figures 8-11.

At the height $z / H=0.8$, we determined a significant acceleration of the longitudinal flow along the left and right edge of the focused street canyon (figure 8). These areas were situated near the street intersection with the street oriented as the approach flow. The channeling effect arose there. In case of the vertical velocity, we detected an area of significant descending and ascending flow along windward and leeward walls, respectively (figure 10). This structure of flow was caused by the horizontal vortex formed within the street canyon. At the level $z / H=1.2$, higher longitudinal velocity was measured above the street canyons oriented in parallel with the approach flow (figure 9). The vertical velocity was not significantly influenced by buildings at that height unlike the longitudinal component (figure 11).

The LES corresponded well with the wind tunnel results of velocity, compare right parts of the figures 8-11. In case of the vertical velocity at the height $z / H=0.8$, a slightly different structure with underestimated magnitudes was observed in LES results (figure 10).

\subsection{Concentration fields}

Values of dimensionless concentration were also distributed approximately symmetrically at both heights, see figures 12 and 13. Significant values were obtained only within the focused street canyon and its close surrounding.

Maximum of concentration was found along the leeward wall of the focused street canyon at the height $z / H=0.8$ (figure 12). A marked decrease of pollutant concentration was observed at height $z / H=1.2$ compared to the previous case. Above the street canyon, the contaminant was detected mainly in the middle part above the roof of the windward building (figure 12).

At the level $z / H=0.8$, LES significantly overestimated pollution concentration in the focused street canyon. In comparison of magnitudes, values computed by LES exceeded results from the wind tunnel twice. In case of level $z / H=1.2$, LES and wind tunnel gave similar values.

\subsection{Advective scalar flux fields}

Considering the advective pollution transport in vertical direction, strong upward pollution transport was found along the leeward wall of the focused street canyon at the lower level $z / H=0.8$. Along the windward wall, fresh air flowed into the canyon (figure 14). It is obvious that the advective pollution transport is mainly driven by the horizontal vortex formed within the street canyon.

We measured negligible negative flux at plane $z / H=$ 1.2. This was caused by the low vertical velocities and concentrations in this area (figure 15).

The LES and the wind tunnel experiment provided very similar magnitudes and distributions of the vertical pollution flux above the focused street canyon. A slight difference in LES results can be observed along the leeward wall. This was caused by underestimated vertical velocities and overestimated concentrations.

\subsection{Turbulent scalar flux fields}

At the level $z / H=1.2$, vertical turbulent fluxes were detected negligible or slightly positive (figure 17). The maximum of the turbulent flux was situated, as in case of concentrations, in the middle part above the roof of the windward canyon wall.

At $z / H=0.8$, an intensive upward turbulent pollution transport was found along the windward wall of the street canyon. The fresh air propagated into the canyon along leeward wall (figure figure 16). Compared with the advective flux, the turbulent pollution transport was opposite to the advective within and above the canyon, compare plots in figures 14) and 16). This phenomenon was also described in [21]. However, the magnitude of the turbulent flux was significantly lower then the advective.

\section{Conclusions}

This paper summarizes the ventilation intensity of the street canyon at different heights and compares the wind tunnel experiment with the large eddy simulation.

The model of the idealised urban-area was designed according to the typical Central-European urban buildings arranged into courtyards. For the wind tunnel experiment, we assembled the experimental set-up for simultaneous measurement of the flow velocity and the tracer gas concentration. This set-up was based on Fast-response Flame Ionisation Detector and Laser Doppler Anemometry. The new line source of tracer gas simulating vehicle emissions was designed and built into the model. The large eddy simulation (LES) of the flow and dispersion was realised in the urban-area model to get complex tree dimensional data from the area. The LES and the wind tunnel data were compared in selected grids.

In the study, we focused mainly on the finite street canyon with the line source situated perpendicular to the approach flow. Longitudinal and vertical velocity components and concentration of the tracer gas were obtained from two horizontal planes at different heights above the canyon (at the eaves level and above the roof). Advective and turbulent pollution fluxes were computed from the results.

Within the street canyon, the velocity and concentration data confirmed a well-known behaviour of the flow and dispersion, which is mainly caused by a large horizontal 
vortex. An intensive advective pollution flux was detected in the lower measured plane. As we expected, the maximum and minimum of the advective flux vas oriented along the leeward wall and windward wall, respectively.

The turbulent pollution transport was smaller compared to the advective one. On the other hand, it still played a significant role in the pollution transport. In both planes, the turbulent pollution transport was opposite to the advective and reached approximately the same magnitudes.

The wind tunnel data corresponded well with the LES above the buildings, however, the results slightly differed within the canyon. We got a different pattern in the vertical velocity field with smaller magnitudes. In the matter of concentration, the LES overestimated the concentration maxima. Nonetheless, the mentioned differences reached acceptable rate, and flow and dispersion were satisfactorily comparable.

In the future, we will investigate the impact of building geometry and orientation on pollution ventilation using both the LES and the wind tunnel modelling.

\section{Acknowledgement}

The authors kindly thank the Charles University in Prague (project GAUK No. 535412) and the Czech Science Foundation (project GAP101/12/1554) for financing this project. This work was also carried out with institutional support RVO: 61388998.

\section{References}

1. J. Fenger, Atmos. Environ. 33, 4877 (1999)

2. G. Hoek, B. Brunekreef, A. Verhoeff, J.V. Wijnen, P. Fischer, Journal of the Air \& Waste Management Association 50, 1380 (2000)

3. P. Kastner-Klein, R. Berkowicz, R. Britter, Meteorology and Atmospheric Physics 87 (2004)

4. K. Ahmad, M. Khare, K. Chaudhry, Journal of Wind Engineering and Industrial Aerodynamics 93, 697 (2005)

5. M. Carpentieri, Reviews in Environmental Science and Bio/Technology pp. 5-8 (2013)

6. P. Salizzoni, L. Soulhac, P. Mejean, Atmospheric Environment 43, 5056 (2009)

7. M. Carpentieri, A.G. Robins, Boundary-Layer Meteorology 135, $229(2010)$

8. M. Carpentieri, P. Hayden, A.G. Robins, Atmospheric Environment 46, 669 (2012)

9. X. Xie, Z. Huang, J. Wang, Atmospheric Environment (2005)

10. L. Kukačka, v. Nosek, R. Kellnerová, K. Jurčáková, Z. Jaňour, The Scientific World Journal 2012, 381357p.1 (2012)

11. L. Kukačka, v. Nosek, R. Kellnerová, K. Jurčáková, Z. Jaňour, EPJ Web of Conferences 45, 01053p.1 (2013)

12. D. Contini, P. Hayden, A. Robins, Atmospheric Environment 40, 7842 (2006)

13. V. Fuka, J. Brechler, Finite Volumes for Complex Applications VI - Problems \& Perspectives (2011)

14. W. Hundsdorfer, B. Koren, M. VanLoon, J. Verwer, Journal of Computational Physics 117, 35 (1995)

15. O.V. Vasilyev, T.S. Lund, P. Moin, Journal of Computational Physics 146, 82 (1998)

16. F. Nicoud, H.B. Toda, O. Cabrit, S. Bose, J. Lee, Physics of Fluids 23, 085106 (2011)
17. VDI Verein Deutscher Ingenieure, ed., Physical modelling of flow and dispersion processes in the atmospheric boundary layer - application of wind tunnels (VDI Verein Deutscher Ingenieure, Düsseldorf, 2000)

18. A.A. Townsend, A Structure of Turbulent Shear Flow (Cambridge University Press, New York, 1976)

19. R.N. Meroney, M. Pavageau, S. Rafailidis, M. Schatzmann, J. Wind. Eng. Ind. Aerodyn. 62, 37 (1996)

20. M. Pavageau, M. Schatzmann, Atmos. Environ. 33, 3961 (1999)

21. K. Bezpalcová, M. Ohba, Z. Jaňour, ICUC-7, Yokohama, Japan, A5-4 pp. 3-6 (2009) 


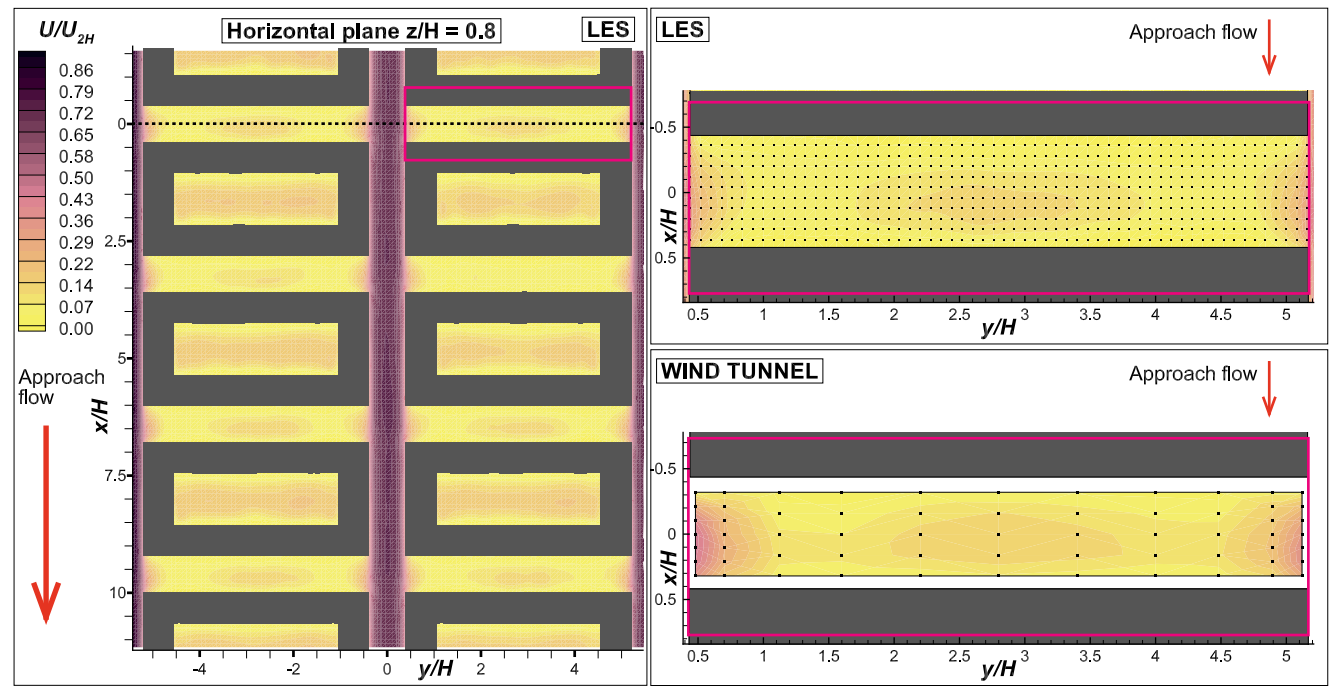

Fig. 8. Mean dimensionless longitudinal velocity $U / U_{2 H}$ at height $z / H=0.8$.
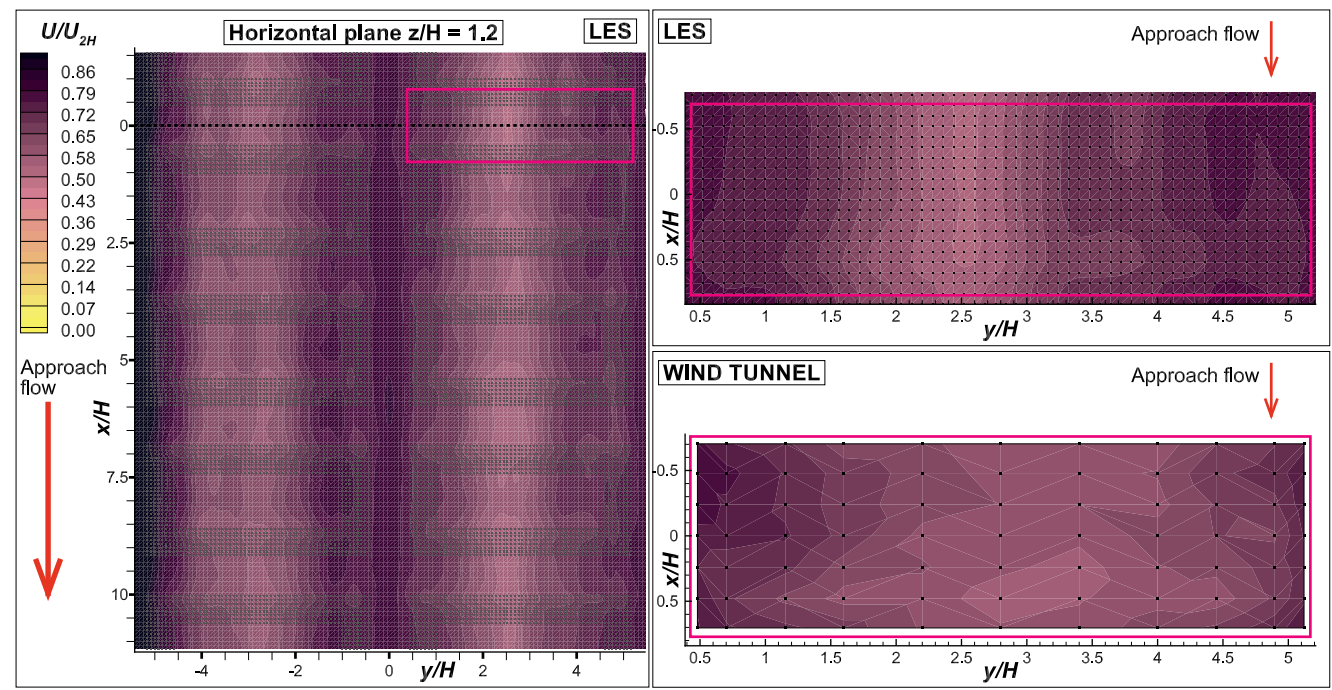

Fig. 9. Mean dimensionless longitudinal velocity $U / U_{2 H}$ at height $z / h=1.2$.
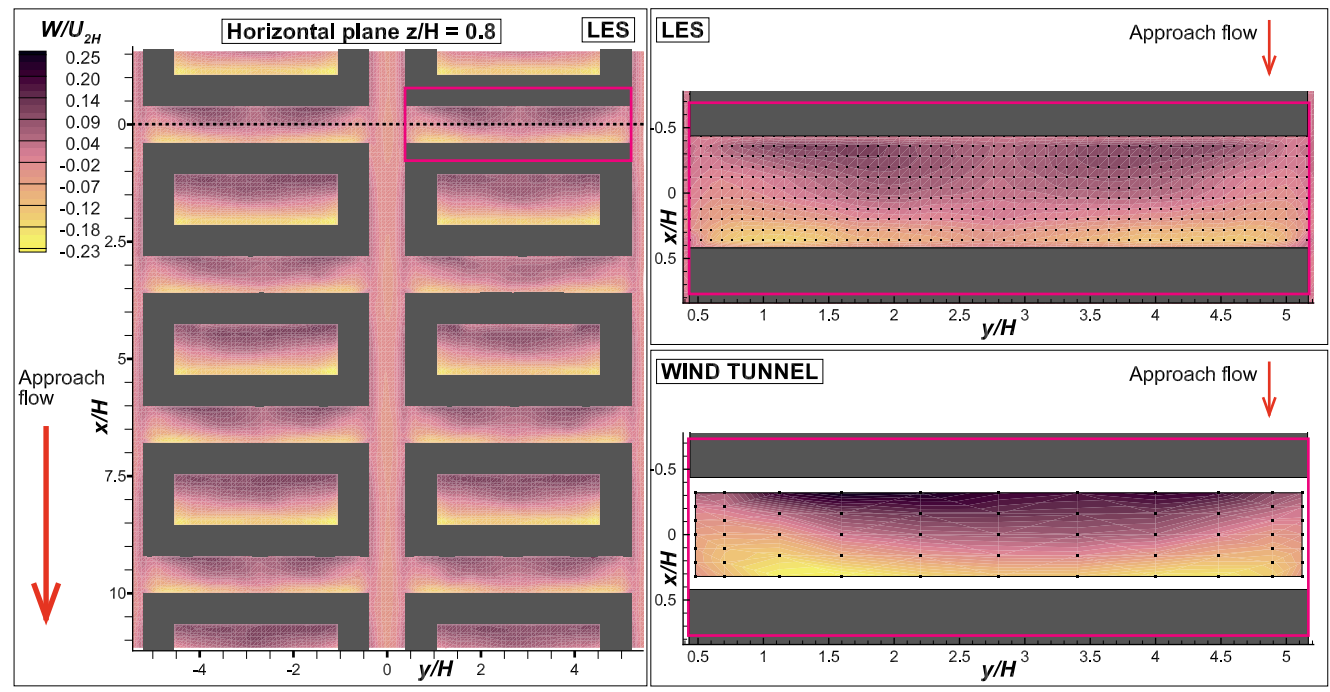

Fig. 10. Mean dimensionless vertical velocity $W / U_{2 H}$ at height $z / H=0.8$. 


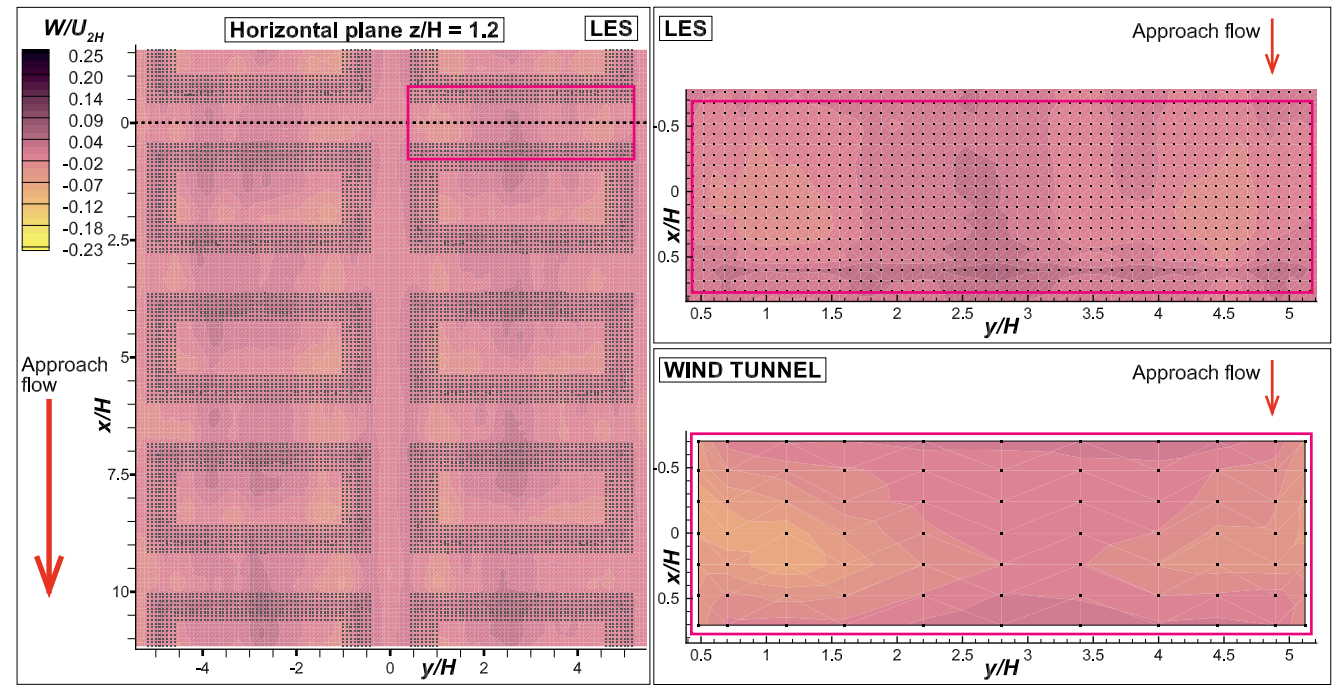

Fig. 11. Mean dimensionless longitudinal velocity $W / U_{2 H}$ at height $z / H=1.8$.

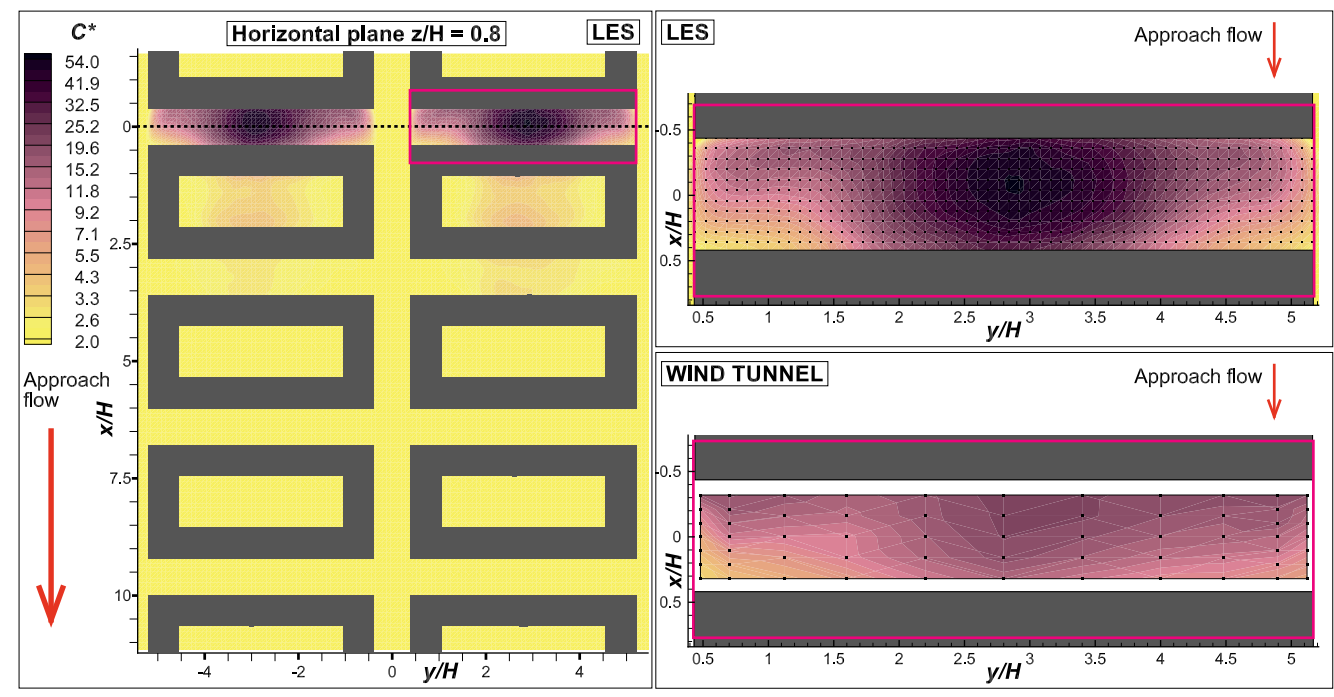

Fig. 12. Mean dimensionless concentration $C^{*}$ at height $z / H=0.8$.
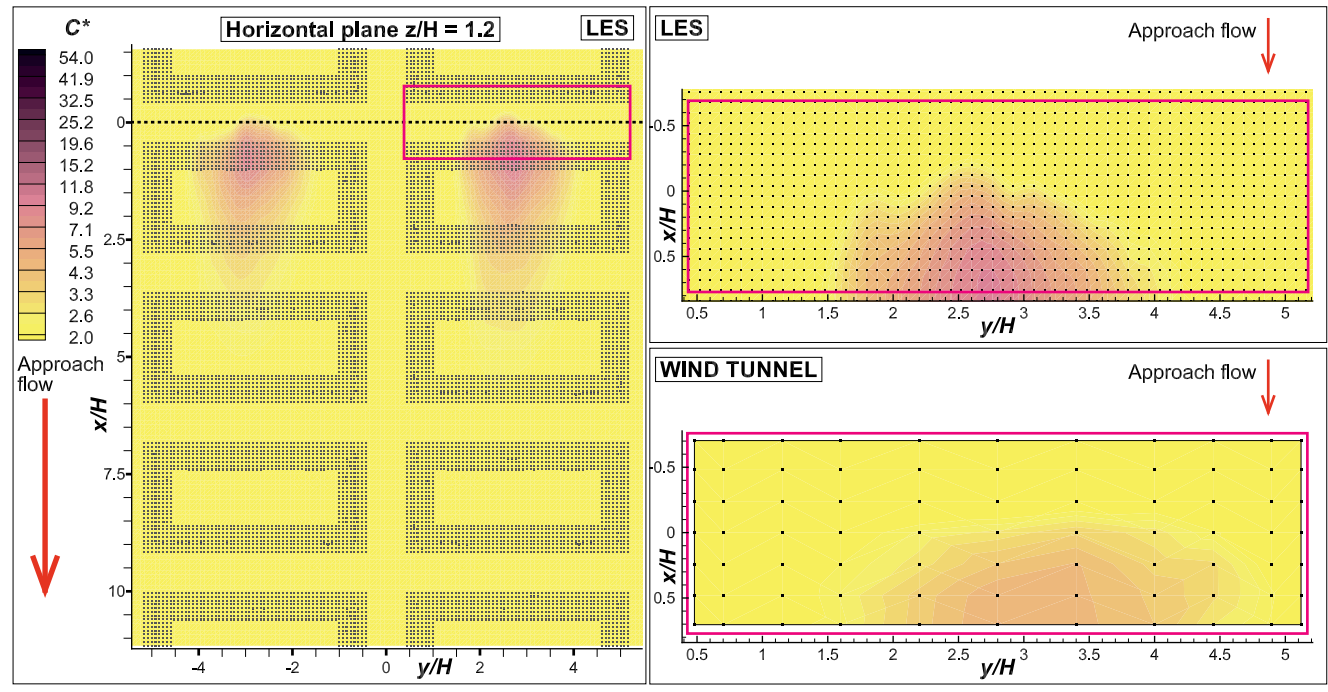

Fig. 13. Mean dimensionless concentration $C^{*}$ at height $z / H=1.2$. 


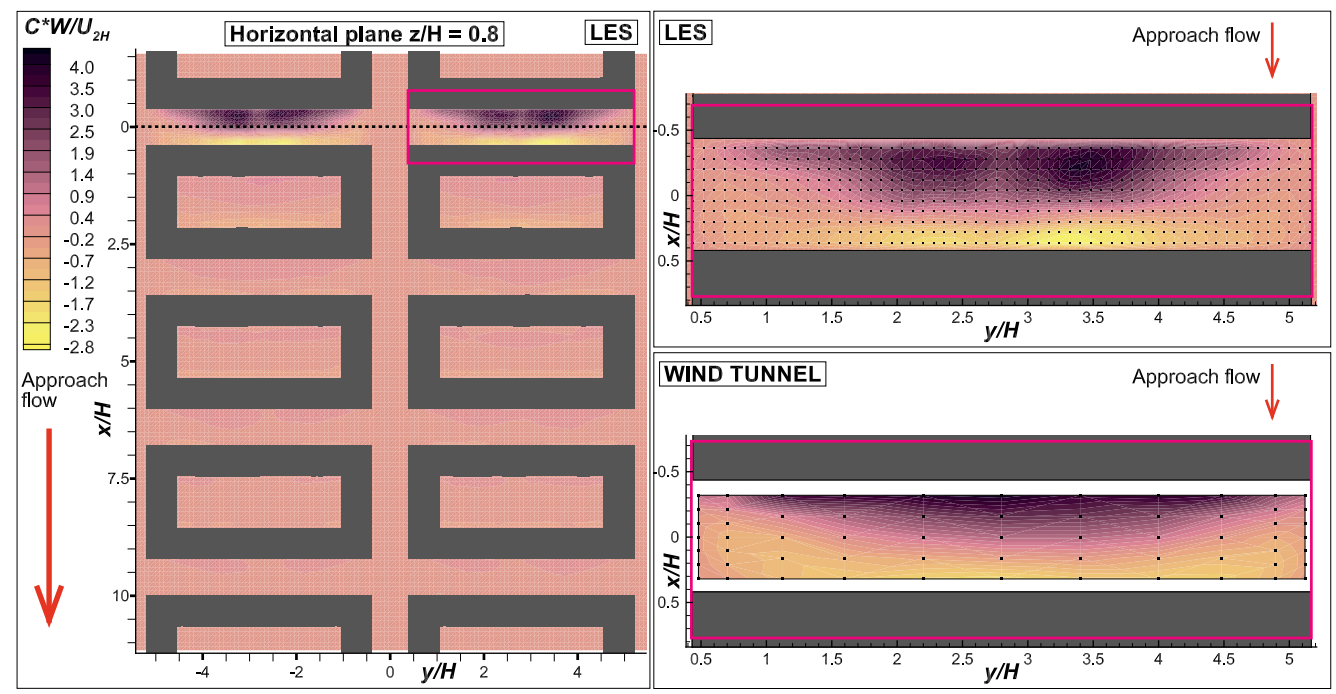

Fig. 14. Vertical dimensionless pollution flux $C^{*} W$ at height $z / H=0.8$.

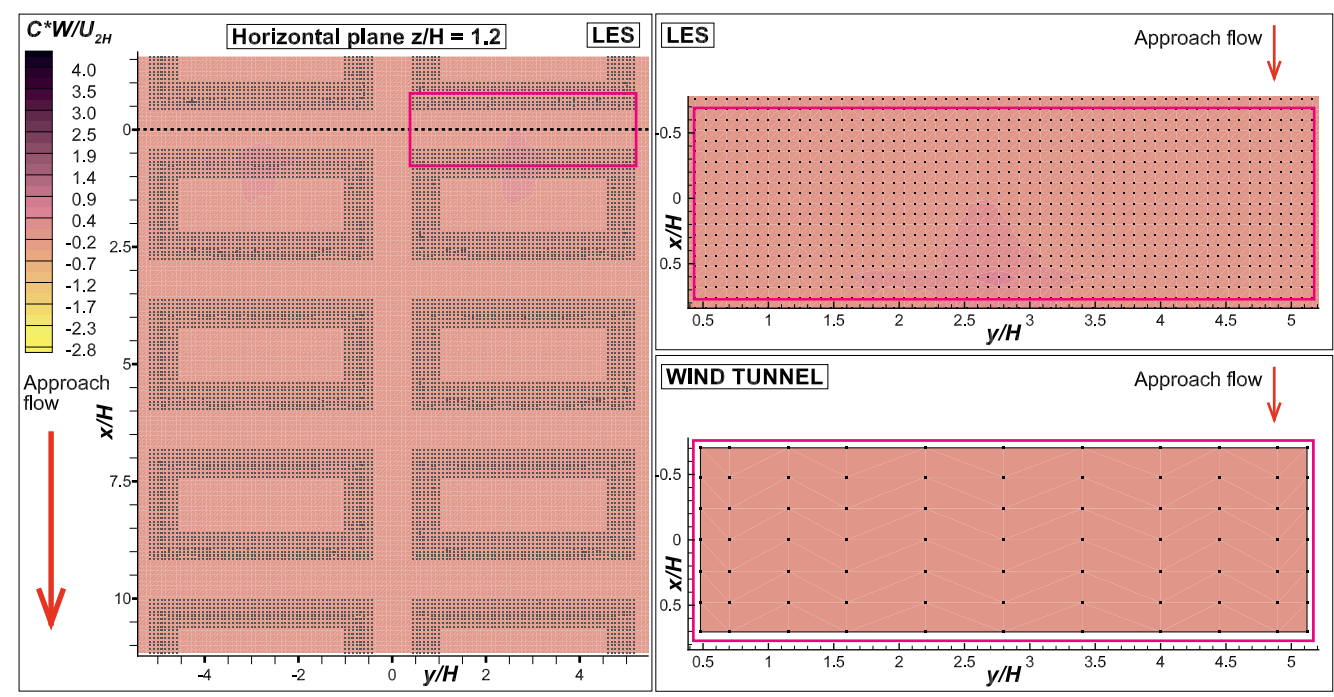

Fig. 15. Vertical dimensionless pollution flux $C^{*} W$ at height $z / H=1.2$.

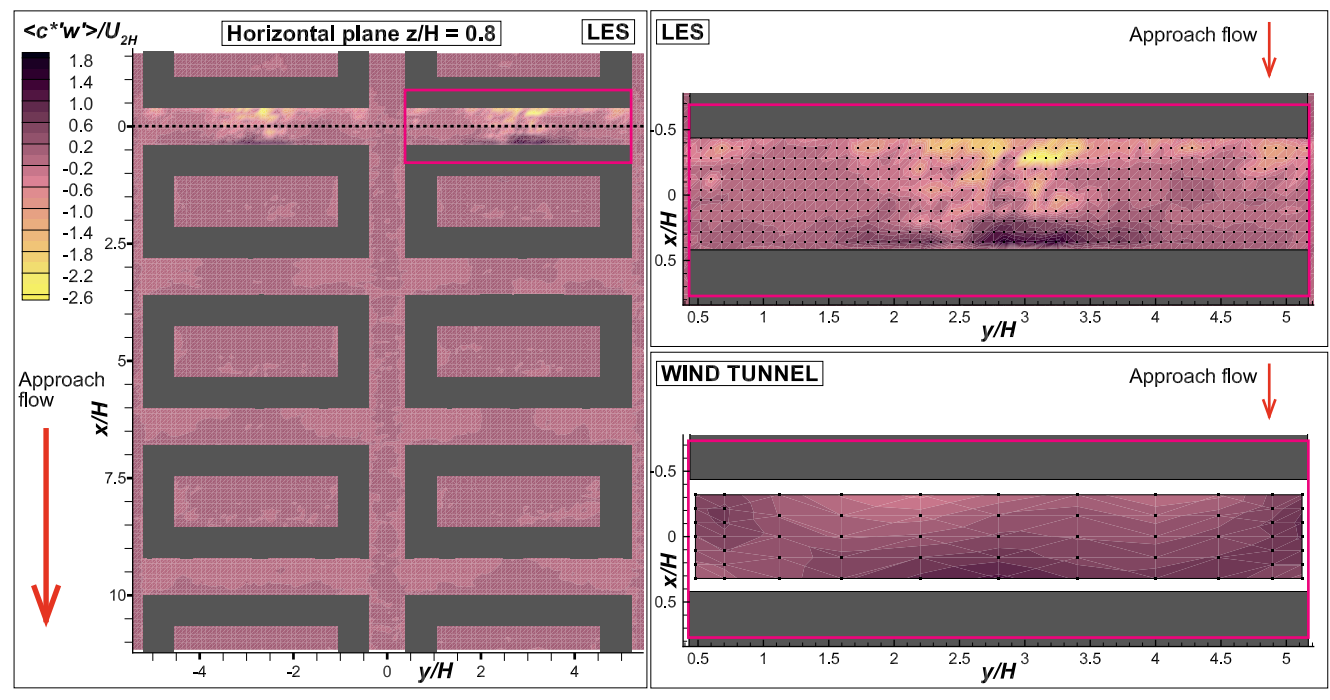

Fig. 16. Vertical dimensionless turbulent flux $c^{*^{\prime}} w^{\prime}$ at height $z / H=0.8$. 


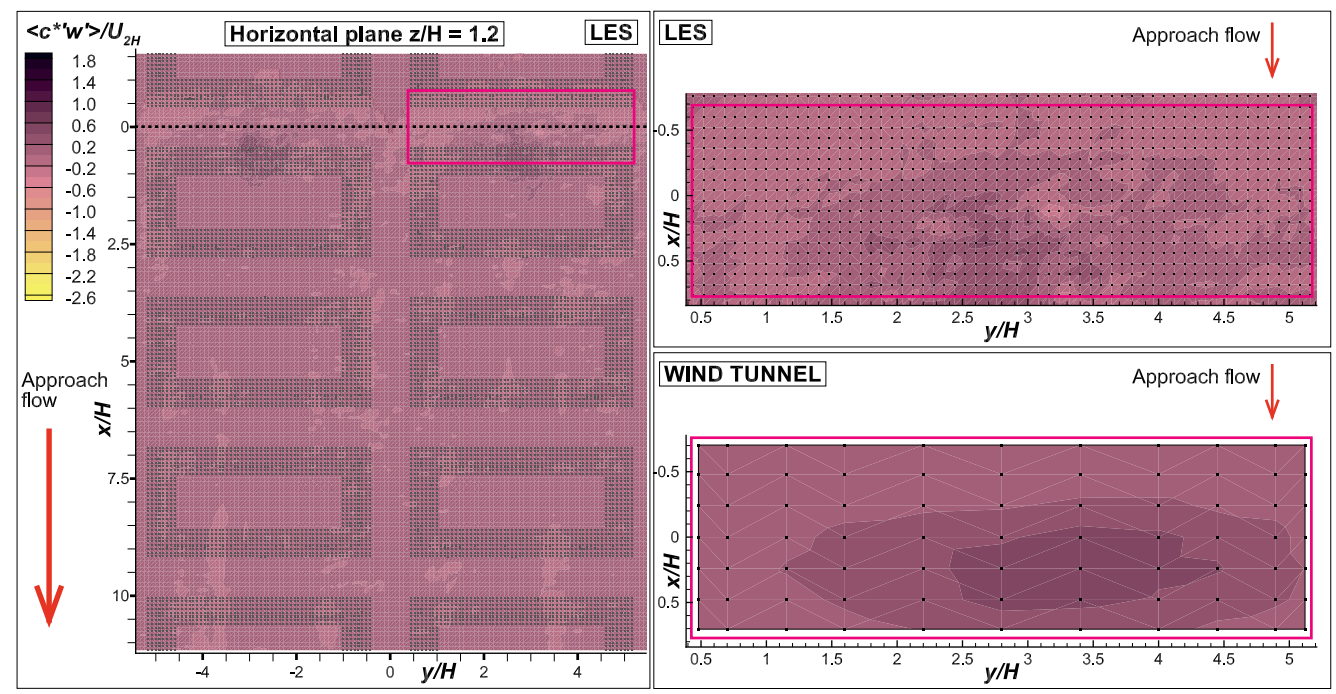

Fig. 17. Vertical dimensionless turbulent flux $c^{*^{\prime}} w^{\prime}$ at height $z / H=1.2$. 